\title{
Low Complexity Channel Estimation for MC-CDMA System Based Chaos under Fast Multipath Channel
}

\author{
Maha George Zia \\ Asst., Prof in Software Engineering Department, College of Engineering, Salahaddin University, Erbil, Iraq
}

\begin{abstract}
Based on interpolation methods, a low complexity channel estimation method for MC-CDMA system based chaos under fast fading multipath channel is proposed.Different interpolation methods are used; Linear interpolation (LI), cubic spline interpolation (CSI), and piecewise cubic Hermite interpolation (PCHI). The multi-carrier scheme is established by using a chaotic generator with different initial conditions, while the pilots are established using another chaotic generator with another different initial conditions. The proposed channel estimator without and with interpolation methods for single user and multiusers is tested under fast frequency selective Rayleigh fading channel. Simulation results demonstrate that the channel estimation of MC-CDMA system based chaos using interpolation methods and when the pilots are chaos signal too, achieves better performance improvement in fast multipath environment compared to the conventional MC-CDMA system when the pilots are considered as WalshHadamard codes. The proposed system is also built and tested using Simulink program.
\end{abstract}

Keywords: chaos based spread spectrum, chaos generator, MC-CDMA system, multicarrier system,interpolation,spreading codes, Walsh-Hadamard codes.

\section{INTRODUCTION}

Recently, multi-carrier code division multiple access (MC-CDMA), which uses a number of low rate orthogonal sub-carriers to reduce the intersymbol interference (ISI) resulting from frequency selective channel, has been attracting much attention [1]. In MC-CDMA system, the channel transfer function of radio channel is usually frequency selective and time variant. Therefore, a dynamic estimation of the channel is necessary for demodulation of MC-CDMA signals [1].

In [2], blind channel estimation for MC-CDMA systems using aperiodic spreading codes was studied. The multiuser parameter estimation problem was solved using relatively low complexity. Since the method is a blind estimation method, it possesses the simplicity essential for practical implementation of MC-CDMA technologies on uplink.

The channel estimation of a multi-carrier code-division multiple access (MC-CDMA) system based on least square (LS) algorithm and linear interpolation in frequency domain over multipath fading channels was proposed in [3]. Different detection receivers for MC-CDMA were also introduced; their algorithms include maximum ratio combining (MRC), minimum mean square error (MMSE), parallel interference cancellation (PIC), and interference cancellation based on expectation-maximization (EM) algorithm. It was proved in [3] that MMSE algorithm provides significant performance gain compared to that with MRC algorithm and the system performance can be further improved by using PIC and EM algorithms.

In [4], performance of a MC-CDMA system employing MMSE multiuser detection (MUD) at receiver, was evaluated using two pilot symbol assisted (PSA) channelestimation schemes: the maximum likelihood (ML) estimation and the MMSE estimation. It was shown that MMSE estimator significantly outperforms the ML estimator in non-sample-spaced channels where path delays are closely spaced with respect to the time resolution of the system.

A cyclic pilot assisted channel estimation technique (CPACE) suitable for MC-CDMA using overlap Frequency-domain equalization (FDE) was proposed in [5]. It was proved that FDE based on the MMSE criterion can take advantage of the channel frequency-selectivity and improve the average bit error rate (BER) performance due to frequency diversity gain. Overlap FDE technique has been proposed that requires no GI insertion and the MC-CDMA using overlap FDE can provide almost the same BER performance as the conventional MC-CDMA downlink using the GI insertion.

The objective of this paper is to design a low complexity channel estimation method for MC-CDMA system based chaos under fast fading environment.Both the multicarrier scheme and pilot's insertion are based on chaos signals generated from two different chaos generators.Least square (LS)channel estimator is used with interpolation methods. The interpolation methods are: linear interpolation (LI), piecewise cubic Hermite interpolation (PCHI) and cubic spline interpolation (CSI).In comb type pilot channel estimation [6], the pilots were inserted within a fixed distance between the symbols while in this research; the pilots (chaos based) are 
inserted randomly between the symbols using random interleaver. The symbol error rate (SER) performance of the proposed channel estimation method is realized for single user and multi-users in 3-paths fast frequency selective Rayleigh fading channel without and with different interpolation methods. Simulation results demonstrate that the performance for the proposed channel estimation based chaos achieves good performance under fast fading channel with and without interpolation methods compared with pilot channel estimation in $[3,6]$ for conventional MC-CDMA that uses Walsh-Hadamard codes for spreading and pilot sequences. The proposed system is also build and tested using Simulink program.

This paper is organised as follows, section II demonstrate the mathematical concepts of interpolation, and section III presents the system description. In section IV, performance evaluation is illustrated using MATLAB program with the proposed system build and tested using Simulink program.Finally, Conclusion is presented in section V.

\section{INTERPOLATION}

Interpolation is the process of defining a function that takes on specified values at specified points. Any two points in the plane, $\left(x_{1}, y_{1}\right)$ and $\left(x_{2}, y_{2}\right)$, with $x_{1} \neq x_{2}$, determine a unique first-degree polynomial in $x$ whose graph passes through the two points. There are many different formulas for the polynomial, but they all lead to the same straight line graph [7].

Given $n$ points in the plane, $\left(x_{i} ; y_{i}\right), i=1, \ldots, n$, with distinct $x_{i}$ 's, there is a unique polynomial in $x$ of degree less than $n$ whose graph passes through the points. This polynomial is called the interpolatingpolynomial because it exactly reproduces the given data [7];

$$
p_{n}\left(x_{i}\right)=\left(y_{i}\right) ; i=1, \ldots, n
$$

\subsection{Linear Interpolation (LI)}

To generalize the concept of linear interpolation [8], let $n \geq 1$, and suppose that $x_{i}, i=0,1, \ldots, n$, are distinctreal numbers (i.e., $x_{i} \neq x_{j}$ for $i \neq j$ ) and $y_{i}, i=0,1, \ldots, n$, are real numbers; we wish to find $p_{n} \in P_{n}$ such that $p_{n}\left(x_{i}\right)=y_{i}, i=0,1, \ldots, n$.

Lemma 2.1.1: Suppose that $n \geq 1$. There exist polynomials $L_{k} \in P_{n}, k=0,1, \ldots, n$, such that [8];

$$
L_{k}\left(x_{i}\right)=\left\{\begin{array}{cc}
1, & i=k \\
0, & i \neq k
\end{array}\right\}
$$

for all $i, k=0,1, \ldots, n$. Moreover;

$$
p_{n}(x)=\sum_{k=0}^{n} L_{k}(x) y_{k}
$$

satisfies the above interpolation conditions; in other words, $p_{n} \in P_{n}$ such that $p_{n}\left(x_{i}\right)=y_{i}, i=$ $0,1, \ldots, n$.

Proof: For each fixed $k, 0 \leq k \leq n, L_{k}$ is required to have nzeros $x_{i}, i=0,1, \ldots, n, i \neq k$ thus, $L_{k}(x)$ is of the form;

$L_{k}(x)=C_{k} \prod_{\substack{i=0 \\ i \neq k}}^{n}\left(x-x_{i}\right)$

Where, $C_{k} \in R$ is a constant to be determined. It is easy to find the value of $C_{k}$ by recalling that $L_{k}\left(x_{k}\right)=1$; using this in (4) yields;

$$
\begin{aligned}
& C_{k}=\prod_{\substack{i=0 \\
i \neq k}}^{n} \frac{1}{x_{k}-x_{i}} \\
& L_{k}(x)=\prod_{\substack{i=0 \\
\text { Inserting (5) into (4), yield; } \\
i \neq k}}^{n} \frac{x-x_{i}}{x_{k}-x_{i}}
\end{aligned}
$$

As the function $p_{n}$ defined by (3) is a linear combination of the polynomials $L_{k} \in P_{n}, k=0,1, \ldots, n$, also $p_{n} \in P_{n}$. Finally, $p_{n}\left(x_{i}\right)=y_{i}, i=0,1, \ldots, n$ is a trivial consequence of using (2) in (3).The interpolating polynomial is easily described once the form of $L_{k}(x)$ is known. This polynomial, called the Lagrange interpolating polynomial in which the linear interpolation is based on [8]. 


\subsection{Piecewise Cubic Hermite Interpolation}

Many of the most effective interpolation techniques are based on piecewise cubic polynomials. Let $h_{k}$ denote the length of the $k$ th subinterval [7];

$$
h_{k}=x_{k+1}-x_{k}
$$

Then the first divided difference, $\delta_{k}$, is given by;

$$
\delta_{k}=\frac{y_{k+1}-y_{k}}{h_{k}}
$$

Let $d_{k}$ denote the slope of the interpolant at $x_{k}$;

$d_{k}=P^{\prime}\left(x_{k}\right)$ interpolants.

For the piecewise linear interpolant, $d_{k}=\delta_{k-1}$ or $\delta_{k}$, but this is not necessarily true for higher order

Consider the following function on the interval $x_{k}<x<x_{k+1}$ expressed in terms of local variables $s=x-x_{k}$ and $h=h_{k}[7]$

$P(x)=\frac{3 h s^{2}-2 s^{3}}{h^{3}} y_{k+1}+\frac{h^{3}-3 h s^{2}+2 s^{3}}{h^{3}} y_{k}+\frac{s^{2}(s-h)}{h^{2}} d_{k+1}+\frac{s(s-h)^{2}}{h^{2}} d_{k}$

This is a cubic polynomial in $s$, and hence in $x$, that satisfies four interpolation conditions, two on function values and two on the possibly unknown derivative values;

$P\left(x_{k}\right)=y_{k}, P\left(x_{k+1}\right)=y_{k+1}$

$P^{\prime}\left(x_{k}\right)=d_{k}, \quad P^{\prime}\left(x_{k+1}\right)=d_{k+1}$

Functions that satisfy interpolation conditions on derivatives are known asHermiteinterpolants.

If both function values and first derivative values at a set of data points are known, then piecewise cubic Hermite interpolation can reproduce those data. But if the derivative values were not given, then the idea is to determine the slopes $d_{k}$ so that the function values do not overshoot the data values, at least locally [7].

If $\delta_{k}$ and $\delta_{k-1}$ have opposite signs or if either of them is zero, then $x_{k}$ is a discrete local minimum or maximum, so the value of $d_{k}=0$.

If $\delta_{k}$ and $\delta_{k-1}$ have the same sign and the two intervals have the same length, then $d_{k}$ is taken to be the harmonic mean of the two discrete slopes;

$\frac{1}{d_{k}}=\frac{1}{2}\left(\frac{1}{\delta_{k-1}}+\frac{1}{\delta_{k}}\right)$

In other words, at the breakpoint, the reciprocal slope of the Hermiteinterpolant is the average of the reciprocal slopes of the piecewise linear interpolant on eitherside.

If $\delta_{k}$ and $\delta_{k-1}$ have the same sign, but the two intervals have different lengths, then $d_{k}$ is a weighted harmonic mean, with weights determined by the lengths of the two intervals;

$$
\begin{aligned}
& \frac{w_{1}+w_{2}}{d_{k}}=\left(\frac{w_{1}}{\delta_{k-1}}+\frac{w_{2}}{\delta_{k}}\right) \\
& \text { Where, } w_{1}=2 h_{k}+h_{k-1} \text { and } w_{2}=h_{k}+2 h_{k-1}
\end{aligned}
$$

\subsection{Cubic spline interpolation}

In fact, spline interpolants are also piecewise cubic Hermite interpolating polynomials, but with different slopes [7].The mathematical spline must have a continuous second derivative and satisfy the same interpolation constraints. The breakpoints of a spline are also referred to as its knots.

The first derivative $P^{\prime}(x)$ of our piecewise cubic function is defined by different formulas on either side of a knot $x_{k}$. Both formulas yield the same value $d_{k}$ at the knots, so $P^{\prime}(x)$ is continuous [7].On the $k$ th subinterval, the second derivative is a linear function of $s=x-x_{k} \quad$ [7];

$$
\begin{aligned}
& p^{\prime \prime}(x)=\frac{(6 h-12 s) \delta_{k}+(6 s-2 h) d_{k+1}+(6 s-4 h) d_{k}}{h^{2}} \\
& \text { If } x=x_{k}, s=0, \text { and } \\
& p^{\prime \prime}\left(x_{k}+\right)=\frac{6 \delta_{k}-2 d_{k+1}-4 d_{k}}{h_{k}}
\end{aligned}
$$

The plus sign in $x_{k}+$ indicates that this is a one-sided derivative.If $x=x_{k+1}, s=h_{k}$ and $p^{\prime \prime}\left(x_{k}-\right)=\frac{-6 \delta_{k}+4 d_{k+1}+2 d_{k}}{h_{k}}$ knot $x_{k}$

On the $(k-1)$ st interval, $P^{\prime \prime}(x)$ is given by a similar formula involving $\delta_{k-1}, d_{k}$ and $d_{k-1}$. At the

$$
p^{\prime \prime}\left(x_{k+1}-\right)=\frac{-6 \delta_{k-1}+4 d_{k}+2 d_{k-1}}{h_{k-1}}
$$


Requiring $P^{\prime \prime}(x)$ to be continuous at $x=x_{k}$ means that $p^{\prime \prime}\left(x_{k}+\right)=p^{\prime \prime}\left(x_{k}-\right)$. This leads to the condition [7];

$$
h_{k} d_{k-1}+2\left(h_{k-1}+h_{k}\right) d_{k}+h_{k-1} d_{k+1}=3\left(h_{k} \delta_{k-1}+h_{k-1} \delta_{k}\right)
$$

If the knots are equally spaced, so that $h_{k}$ does not depend on $k$, this becomes

$$
d_{k-1}+4 d_{k}+d_{k+1}=3\left(\delta_{k-1}+\delta_{k}\right)
$$

The slopes $d_{k}$ of a spline are closely related to the differences $\delta_{k}$. In the spline case, they are a kind of running average of the $\delta_{k}$ 's [7].

\section{System Description}

Figure (1) illustrates the MC-CDMA with the proposed channel estimation. The proposed method is based on LS estimator and interpolation methods. The interpolation methods used are linear interpolation (LI), piecewise cubic Hermite interpolation (PCHI) and cubic spline interpolation (CSI).

The information data is modulated using QPSK modulator. The data modulated symbols are represented by:

$D(m)=\left[d_{1}, d_{2}, d_{3}, \ldots, d_{M}\right]$,

The user specific scrambling code $c_{v}$ is generated from a chaotic generator by changing its initial conditions and is considered as spreading in frequency. [9];

$C(v)=\left[c_{1}, c_{2}, c_{3} \ldots, c_{v}\right]$

where,

$c_{n}=4\left(c_{n-1}^{2}-3 c_{n-1}\right), n=1,2, \ldots, v$

The spreaded data symbols are converted from serial-to-parallel (s/p) vector $X_{s}$ of length $N_{d}=m \times v$. The pilot vector $X_{p}$ of length $N_{p}$ is represented by;

$X_{p}\left(N_{p}\right)=\left[x_{1}, x_{2}, \ldots, x_{N_{p}}\right]$

Where,

$x_{n}=4 x_{n-1}\left(1-x_{n-1}\right), n=1,2, \ldots, N_{p}$

$X_{p}$ isgenerated by varying the initial conditions using the chaotic generator given in (25), then this vector is concatenated with the vector $X_{s}$ to form a concatenated vector $X_{s p}$ of length $N_{c}$;

$X_{s p}=\left[X_{s} X_{p}\right]$

$N_{c}=N_{p}+N_{d}$ is the number of sub-carriers of inverse fast Fourier transform (IFFT). $X_{s p}$ is input to a random interleaver to form an interleaved vector $X_{s p I}$ of length $N_{c}$. IFFT block is used to transform $X_{s p I}$ to a time domain signal. A guard interval (GI), which is chosen to be larger than the expected delay spread of the channel, is inserted to $X_{s p I}$ to prevent inter-symbol interference. After parallel-to-serial ( $\mathrm{p} / \mathrm{s}$ ) convertor, the transmitted signal will pass through a 3-paths frequency selective Rayleigh fading channel with additive white Gaussian noise (AWGN). The channel is modeled using Jake's model [10] where its discrete impulse response, $h_{n}$ is defined as:

$h_{n}=\sum_{l=1}^{L} h_{l} \operatorname{sinc}\left\{\frac{\tau_{l}}{T_{s}}-n\right\}$

where, $T_{S}$ is the input sample period to the channel, $\tau_{l}$ is the set of path delays, $L$ is the total number of propagation paths, and $h_{l}$ is the $l^{\text {th }}$ path gain.

At the receiver, after removing GI, $(\mathrm{s} / \mathrm{p})$ conversion, the received signal is sent to FFT block which transforms it into a frequency domain vector $\mathrm{Q}(\mathrm{k})$ of length $N_{c}$.

$Q(k)=X_{s p I}(k) H(k)+W(k), \quad k=1.2 .,,,,,,, N_{c}$

Let $X(k)=X_{S p I}$ for simplicity, $H(k)=F F T\{h(n)\}$ and $W(k)=F F T\{A W G N)$. Now $Q(k)$ is deinterleaved, the received pilot signals $\mathrm{P}(\mathrm{k})$ of length $\mathrm{Np}$ are extracted and used to estimate the channel impulse response for the data sub-channels in the channel estimation block . LS channel estimator is used [7, 8, 9]:

$H_{L S}=\frac{P(k)}{X_{P}}$

$H_{L S}$ is interpolated using the interpolation methods which were described in section II to obtain the interpolated channel estimation vector $H_{-}$est $i$. The received data from the deinterleaver, $\mathrm{QQ}(\mathrm{k})$ of length $\mathrm{N}_{\mathrm{d}}$ is now multiplied with $H_{-} e s t_{-} i$ to obtain the estimated data vectors. As shown in Fig.1, the estimated data vectors each of length $\mathrm{N}_{\mathrm{d}}$ are summed, $\mathrm{p} / \mathrm{s}$ converted and applied to a despreader to obtain the estimated output data. 

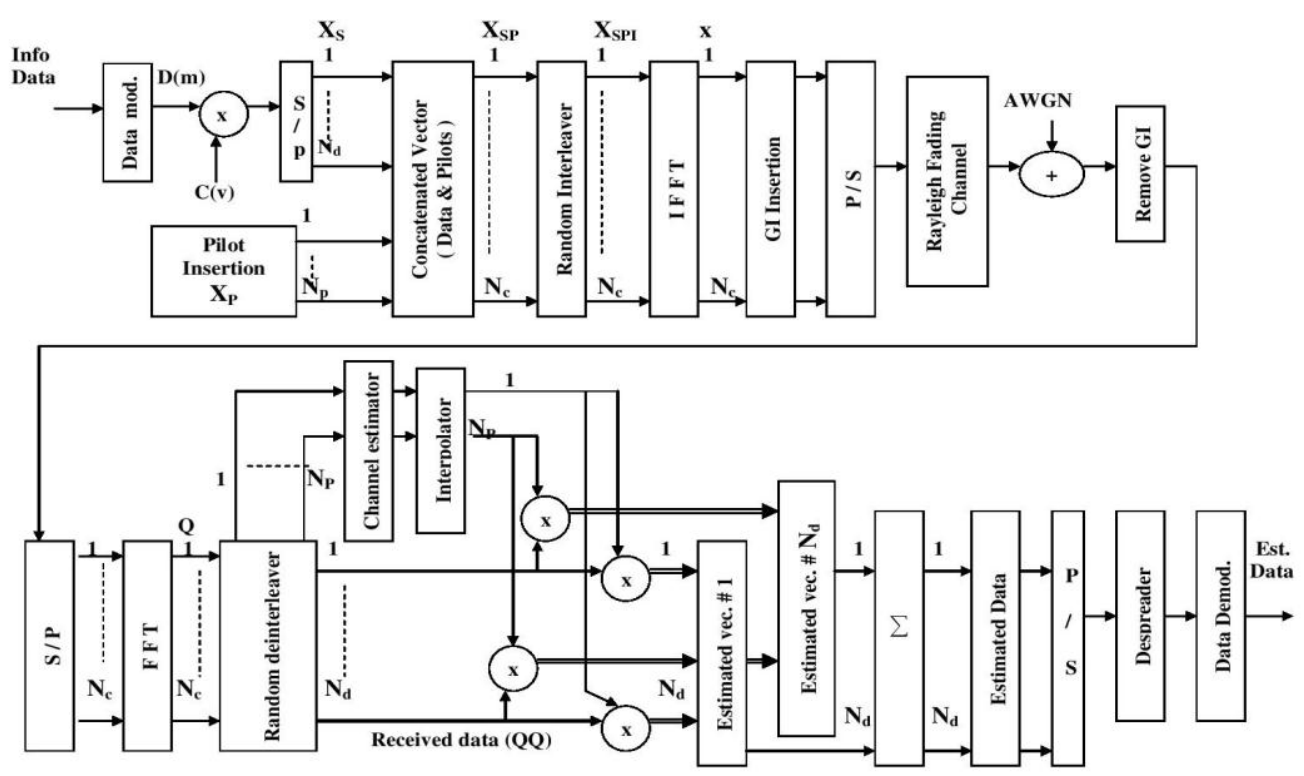

Fig. 1 MC-CDMA system based chaos with proposed channel estimation method

\section{Performance Evalution}

The performances of single user and multi-users ( 8 users) of MC-CDMA system based chaos under fast fading environment with the proposed channel estimation method are evaluated using MATLAB simulation program as shown in Fig.2, Fig. 3, and Fig. 4. The proposed system is also build and tested using Simulink program as shown in Fig. 5. Table 1, list the simulation parameters that are used in this work.

Table.1 Simulation Parameters

\begin{tabular}{|c|c|}
\hline Number of transmitted bits & 100000 \\
\hline Type of modulation & QPSK \\
\hline Spreading factor & Random interleaver \\
\hline Interleaver type & N = 512 \\
\hline Size of FFT and IFFT & 64 \\
\hline Length of Guard insertion (GI) & Chaos sequence, Walsh - \\
\hline Pilot type & 16,32 \\
\hline Pilot numbers & 3 \\
\hline Rayleigh Fading channel paths & $-3-8] \mathrm{dB}$ \\
\hline Rayleigh Fading channel gains & $40 \mathrm{~Hz}$ \\
\hline Doppler Frequency & $(0-30) \mathrm{dB}$ \\
\hline AWGN & 1,8 \\
\hline Number of users & Linear, nearest, pchip, and spline \\
\hline Interpolation types & LS estimator \\
\hline Channel estimation &
\end{tabular}

Figure 2 and Fig.3 illustrate the SER performances of MC-CDMA system based chaos in single user and eight users with proposed channel estimation without interpolation and with three types of interpolation methods. It can be noticed form both figures that SER performance using Pchip interpolation method is nearly the same as spline interpolation method but spline interpolation method is better for single and eight users in frequency selective Rayleigh fading channel under fast fading.

In Fig. 4, SER performance performances of MC-CDMA system based chaos using spline interpolation for eight users is presented. Different types and numbers of pilot signal are used, 16 and 32 as chaos and Walsh- 
Hadamard sequences. It can be seen that as the number of pilots is increased, the SER performances using chaos and Walsh-Hadamard sequences becomes closer.

In Fig. 5, the proposed system is build and tested using Simulink program, the input is represent by a random integer generator frame based, Both the spreading code and pilots which are chaos based are builtas subprograms using MATLAB functions. The LS channel estimation is also build using MATLAB function while the interpolator is represented by FIR interpolator block. Using the parameters which are in Table 1, the proposed system is tested for SNR= 0dB in AWGN and 3-paths fast Rayleigh fading channel where the SER is recorded as 0.3167 .

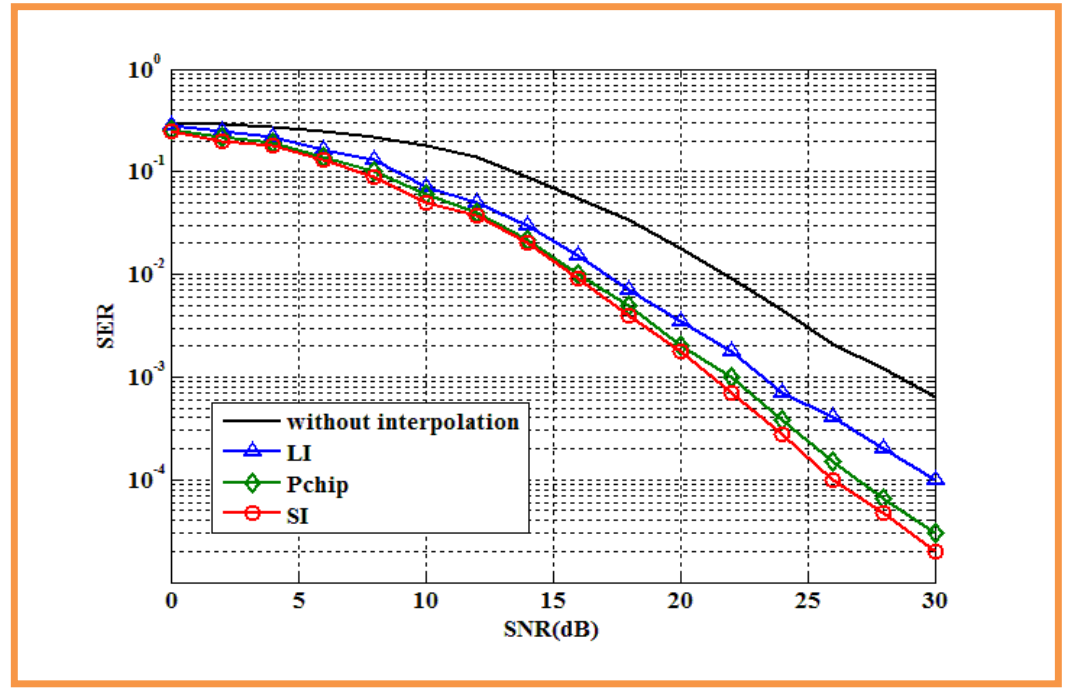

Fig.2 SER performance of MC-CDMA system for one user with and without interpolation

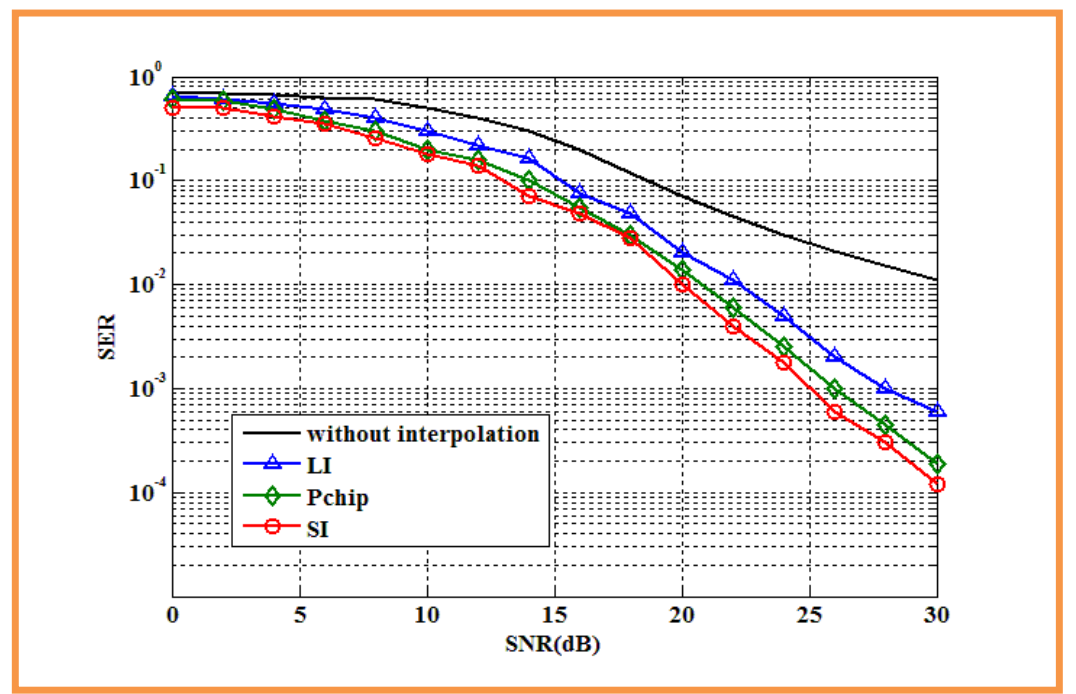

Fig.3 SER performance of MC-CDMA system for eight users with and without interpolation 


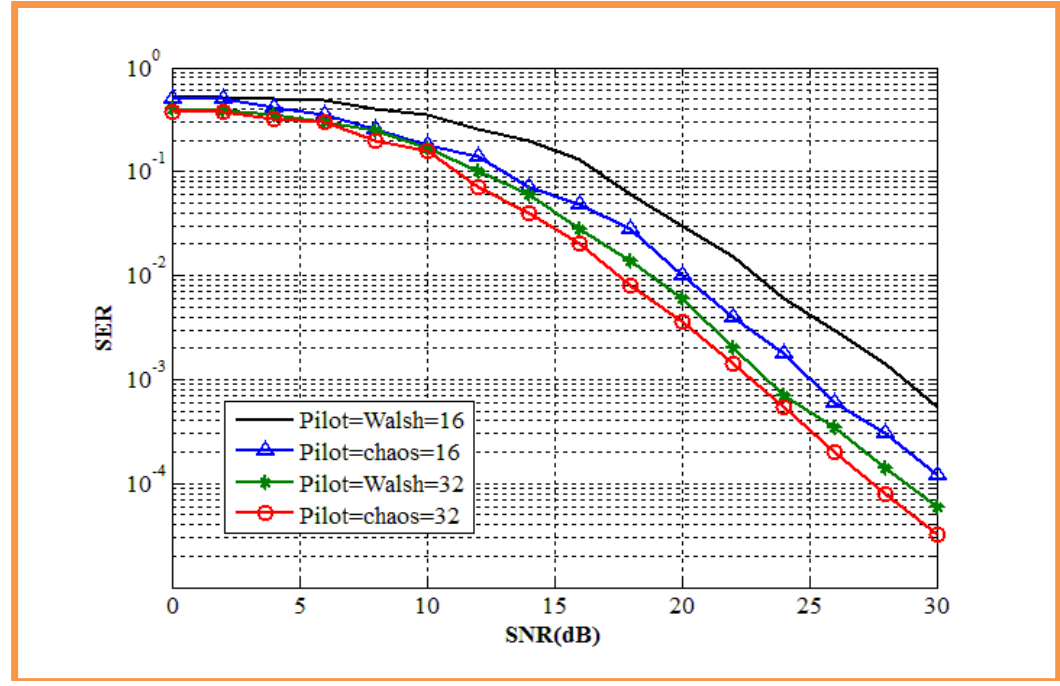

Fig.4 SER performance of MC-CDMA system for eight users with SI and using different pilot types

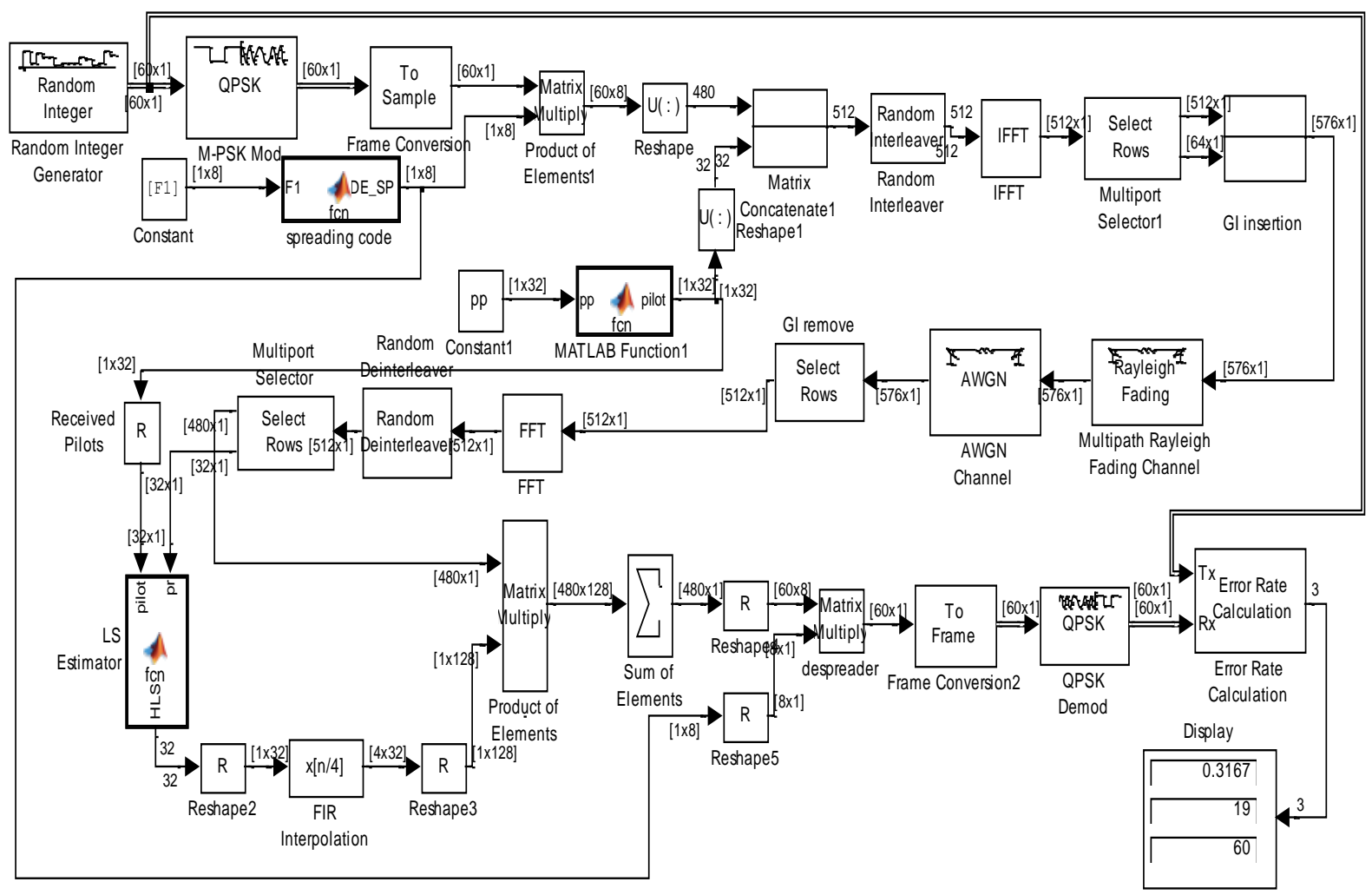

Fig. 5 MC-CDMA system based chaos with proposed channel estimation using Simulink

\section{CONCLUSION}

A low complexity channel estimation method for MC-CDMA system based chaos was designed for single user and multi - users in fast frequency selective Rayleigh fading channel.Both the multicarrier scheme and pilot's insertion were based on chaos signals generated from two different chaos generators. Random interleaverwas used and the proposed method was based on LS channel estimator with interpolator. Three interpolation methods were used. Simulation results demonstrated that the channel estimation of MC-CDMA system using interpolation methods and when the pilots are chaos signal achieves better performance 
improvement compared to the same proposed system when the pilots are considered as Walsh-Hadamard codes (conventional MC-CDMA), but the performance becomes closer as the number of pilots is increased. The proposed system was also built and tested using Simulink program.

\section{REFERENCES}

[1] S. Hara and R. Prasad," Design and performance of Multicarrier CDMA system in frequency-selective Rayleigh fading channels," IEEE Trans. Vehicle. Technology, vol. 48, no. 5, Sep. 1999, pp.1584-1595.

[2] U. Tureli, D. Kivanc and H. Liu," Channel estimation for multicarrier CDMA," ICASSP 2000, 2000, pp. $2909-2912$.

[3] S. Iraji, T. Sipila and J. Lilleberg," Channel estimation and signal detection for MC-CDMA in multipath fading channels," The 14"' IEEE 2003 international Symposium on Personal lndoor and Mobile Radio Communication Proceedings, 2003, pp. 2286-2290.

[4] Trivedi and R. Gupta," Pilot Symbol Assisted Channel Estimation for Uplink MC-CDMA Systems using Parametric Channel Modeling," International Symposium on Communications and Information Technologies (ISCIT 2007), 2007, pp. 989-993.

[5] H. Tomeba, K. Takeda, F. Adachi," Pilot assisted channel estimation for MC-CDMA signal transmission using overlap FDE," ICCS 2008, 2008, pp. $1106-1110$

[6] S. Coleri, M. Ergen, A. Puri, and A. Bahai," Channel Estimation Techniques Based on Pilot Arrangement in OFDM Systems," IEEE Trans. Broadcasting, vol. 48, no. 3, Sept. 2002, pp. 223-229,

[7] www.mathworks.com/moler/interp.pdf

[8] E. Süli, and D. F. Mayers, An Introduction to Numerical Analysis, Cambridge University Press, NY, USA, 2003.

[9] Heidari-Bateni G., and McGillem C.D., "Chaotic Sequences for Spread Spectrum: An Alternative to PN-Sequences", IEEE ICWC '92, 1992, pp. 437-440. 